\title{
Scientific astrophysical payloads for pico and nano-satellites
}

\section{René Hudec ${ }^{1,2 * \dagger}$}

${ }^{1}$ Czech Technical University in Prague, Faculty of Electrical Engineering, Technicka 2, CZ 16000 Prague, Czech Republic

${ }^{2}$ Astronomical Institute of the Academy of Sciences of the Czech Republic Fricova 298 - CZ 25165 Ondrejov, Czech Republic

E-mail: rene.hudec@gmail.com

\section{Vojtěch Šimon ${ }^{1,2 \ddagger}$}

${ }^{1}$ Czech Technical University in Prague, Faculty of Electrical Engineering, Technicka 2, CZ 16000 Prague, Czech Republic

${ }^{2}$ Astronomical Institute of the Academy of Sciences of the Czech Republic Fricova 298 - CZ 25165 Ondrejov, Czech Republic

E-mail: vojtech.simon@gmail.com

\section{Vladimír Tichý $1 \S$}

${ }^{1}$ Czech Technical University in Prague, Faculty of Electrical Engineering, Technicka 2, CZ 16000 Prague, Czech Republic

E-mail: vladimir-tichy@email.cz

The picosatellites, recently in development at many institutes and universities (e.g. Schmidt et al. 2009 and Schilling 2006), require a miniaturized payload. We present and discuss an idea for a suitable scientific payload, namely a miniature Lobster Eye X-ray telescope.

Frontier Research in Astrophysics,

26-31 May 2014

Mondello (Palermo), Italy

* Speaker.

${ }^{\dagger}$ A footnote may follow.

$¥$ A footnote may follow.

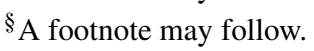




\section{Introduction}

The miniature satellites are recently in development at many institutes and universities, mostly with a participation of students. The fast development of the related techniques and technologies enables to consider small scientific payloads for these satellites. Below we give a small summary of pico (cube) and nanosatellites.

- The pico and nanosatellites are in development at many universities, mostly with involvement of students and education

- CubeSat standard size is 1 Liter Volume, i.e. $10 \times 10 \times 10 \mathrm{~cm}$, and a typical weight is $1.3 \mathrm{~kg}$

- Multiple modules are possible, i.e. $3 \mathrm{U}=3$ modules/units, i.e. $10 \times 10 \times 30 \mathrm{~cm}$, typically up to $12 \mathrm{U}$

- The typical masses are as follows: femtosatellite -10 to $100 \mathrm{~g}$, picosatellite -0.1 to $1 \mathrm{~kg}$, nanosatellite -1 to $10 \mathrm{~kg}$, microsatellite -10 to $100 \mathrm{~kg}$

- Recent technological progress allows to consider a use in astrophysics

\section{Scientific payload for pico and nanosatellite}

In this paper, we focus on application of pico and cubesatellites in high-energy astronomy and astrophysics. Motivation for application of picosatellites in high-energy astrophysics is as follows.

- The recent situation in experimental satellite high-energy astrophysics is not very promising

- LOFT was not selected as ESA M3 mission

- ESA XEUS, NASA Cons X, ESA/NASA/JAXA IXO were canceled

- A hope remains with Athena+ but that is a distant future

- QUESTION: IN WHAT EXTEND MAY THE VERY SMALL SATELLITES FILL THE GAP?

However, there are the following strict requirements for the scientific payload for picosatellites.

- It must fit a small volume, typically $30 \times 10 \times 10 \mathrm{~cm}$ or less ( 3 U, i.e. 3 cubesat modules)

- Low weight, less than $1 \mathrm{~kg}$

- Low power consumption of about 10 Watts or less

- Technological tests: TRW increase, flight demonstration, etc.

- Reasonable science

- Not easy to find such an instrumentation 


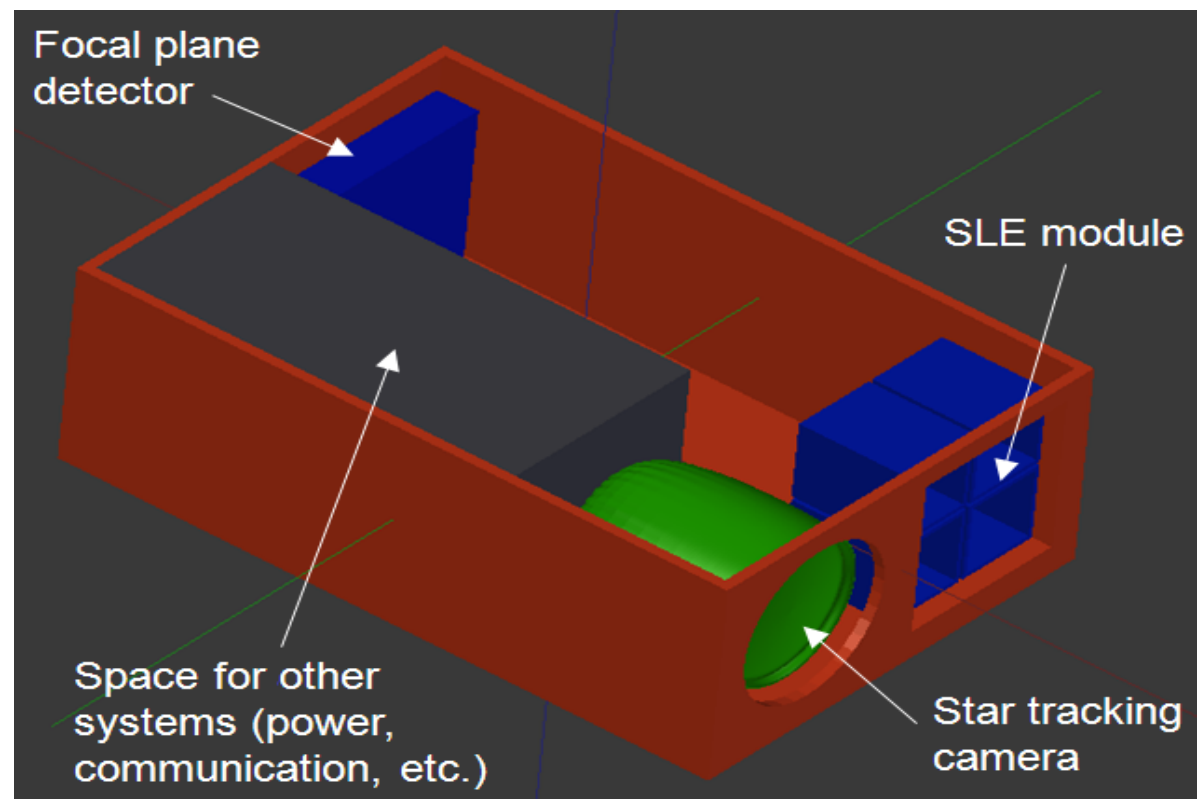

Figure 1: A $6 \mathrm{U}$ cubesatelellite

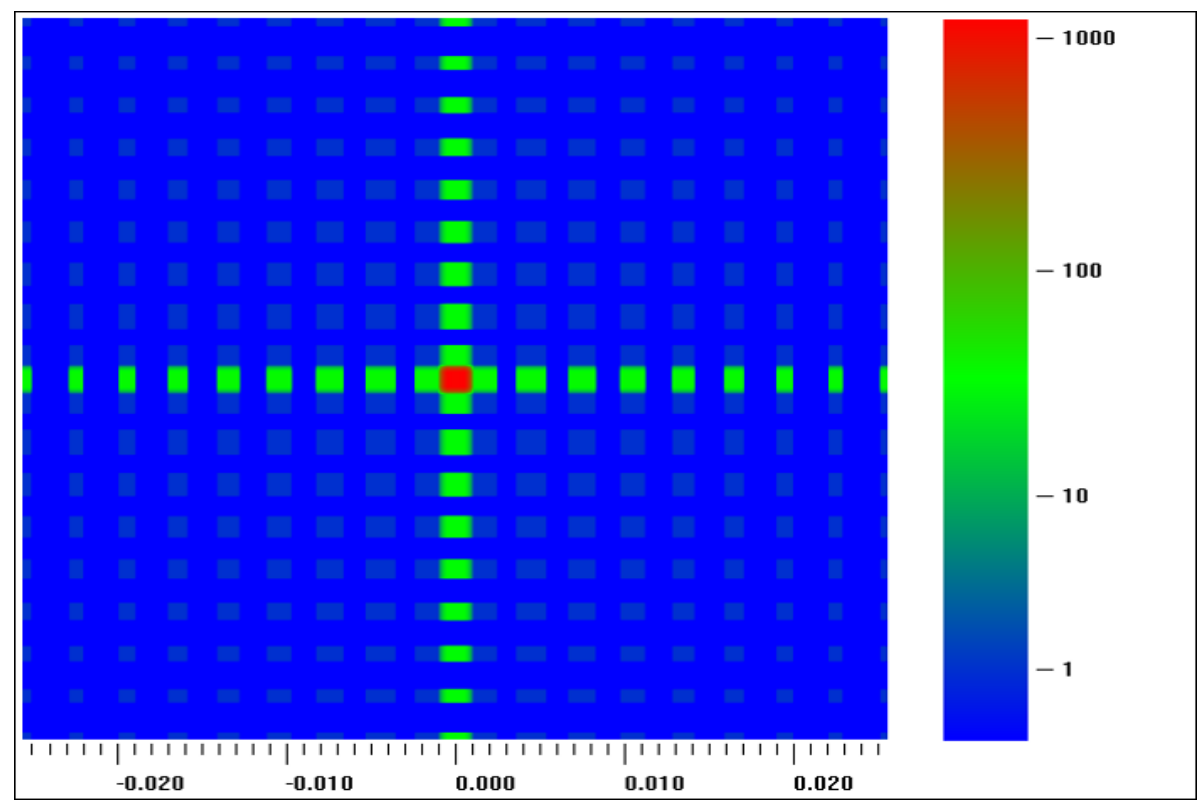

Figure 2: A simulated image of a point-like source in the focal plane, the image was obtained by a simplified ray-tracing algorithm (Tichý 2013b)

The purpose of this paper is to give and discuss idea for picosatellites scientific payload. It is not describing any particular space mission hence no details about the real space mission such as processing resources, ground support system and TTC, orbit, temperature requirements of detectors and their qualification plan, etc., are addressed here. 


\section{Miniature X-ray/telescope - monitor}

The Lobster Eye (LE) X-ray optics was originally proposed by Schmidt (1975) and Angel (1979). Since then, numerous test specimens of Lobster Eye telescopes were designed and tested (e.g. Inneman et al. 1999; Hudec et al. 2000, 2003, 2004; Tichý et al. 2009, 2011). The LobsterEye (LE) X-ray telescope can be miniaturized for an application in picosatellites. The LE telescopes are novel wide field X-ray telescopes with the field of view (FOV) of 100 sq. deg. They are more easily possible (a classical X-ray optics has the FOV of only 1 deg or less) and are based on a real analogy with the lobster eyes.

The LE payload for picosatellites also requires miniaturized focal detectors. The best available option for the author team of this paper is the detector Medipix (Timepix). Medipix is a family of photon counting pixel detectors developed by an international collaboration, hosted by CERN (http://medipix.web.cern.ch/MEDIPIX/). The CTU in Prague is a member of this cooperation. The Medipix detector represents a suitable imaging detector for a use in space LE telescopes, as it is a pixelated photon counting semiconductor detector which features adjustable energy thresholds allowing multispectral X-ray imaging. These detectors offer several different working modes for X-ray imaging applications (e.g. Procz et al., 2012): (i) Single pixel mode (SPM): one threshold with a large 24-bit counter providing a high dynamic range or two thresholds with separate 12 bit counters providing two energy channels. This enables dual channel X-ray imaging with a single acquisition, and (ii) Charge summing mode (CSM): to reduce the influence of charge sharing effects, in this mode charge deposited to adjacent pixels will be summed up and assigned to the pixel featuring the highest signal.

However, the following items are important: 1 . The detector is not yet space qualified; 2 . Its spectral coverage starts only in energies above $3 \mathrm{keV}$, while the spectral coverage of the LE optics in the Schmidt arrangement is typically from the visible light up to the energies of 8 or $10 \mathrm{keV}$.

The LE Telescope for a picosatellite can be represented e.g. by the following example:

- Energy of $4.5 \mathrm{keV}=7.2 \times 10^{-6} \mathrm{erg}$

- Focal length $250 \mathrm{~mm}, \mathrm{~h}=30 \mathrm{~mm}$

- Weight less than $1 \mathrm{~kg}$ (optics: $50 \mathrm{~g}$, detector: $50 \mathrm{~g}$ )

- FOV 2 deg $\times 2$ deg, gain=820

- Daily minimal flux: $9.2 \times 10^{-10} \mathrm{erg} \mathrm{s}^{1} \mathrm{~cm}^{-2}$

However, a more suitable spacecraft is a $6 \mathrm{U}$ CubeSat as it allows to accommodate several LE modules to increase the final FOV (Tichý et al. 2013a). We elaborated a design of a new SLE prototype as follows. More mirror plates: 333 per set, a larger input area $(10 \times 10 \mathrm{~cm})$ and hence higher gain, a special coating to increase the reflectivity at higher energies, a better manufacturing technology, the focal length kept at $250 \mathrm{~mm}$, larger FOV (approximately $10 \times 10$ degrees).

\subsection{Science objectives}

Wide field X-ray monitors of Lobster Eye type were demonstrated to play an important role in modern astrophysics (e.g. Hudec et al. 2007; Švéda et al. 2004). The most important scientific cases are briefly summarized below. 
- A long-term (months) measurement of the light curves of bright persistent X-ray binaries in the direction toward the center of the Galaxy in the soft X-ray band

- Detection and measurement of the light curves of bright transient events of X-ray binaries in the direction toward the center of the Galaxy in the soft X-ray band

\subsection{Modes of Operation}

The LE telescopes can typically serve in two basic operation modes as follows.

- Starrying (pointed) mode - only for satellite with pointing

- Scanning mode (no satellite pointing and/or stabilization required)

\section{Conclusions}

The proposed mission (miniature LE X-ray telescope/monitor in a picosatellite) can acquire scientifically important data for a low price. The optics for the presented mission is feasible. The scientific justification is strong, including several perspective areas of modern astrophysics. A new prototype of the optics (based on the optimized design for a picosatellite) is to be developed and tested.

Acknowledgments This work was supported by the grant GA CR 13-33324S. The scientific part of the study (long term evolution of X-ray sources) is linked to the GA CR grant 13-39464J as well.

\section{References}

[1] Hudec, R., et al., Proc. SPIE 5488, UV and Gamma-Ray Space Telescope Systems, (11 October 2004); doi: 10.1117/12.551915, 2004

[2] Hudec, R., Pína, L., Inneman, A., Švéda, L., LOBSTER - Astrophysics with Lobster Eye Telescopes, in Exploring the Cosmic Frontier, ESO Astrophysics Symposia European Southern Observatory 2007, pp.73-74, 2007

[3] Angel, J. R. P., 1979, Astroph. J., 364, 233

[4] Inneman, A., et al., 2000, Proc. SPIE, 4138, 94

[5] Hudec, R., et al., 2000, SPIE Proc. 4012, 432

[6] Hudec, R., et al., 2003, SPIE Proc. 4851, 578

[7] Hudec, R., et al., 2004a, SPIE Proc. 5488, 449

[8] Hudec, R., et al., 2004b, Nucl. Phys. B Proc. Suppl. 132, 320

[9] Procz, S., et al., Medipix3 CT for material sciences Proc. 14th INTERNATIONAL WORKSHOP ON RADIATION IMAGING DETECTORS, 1âĂŞ5 JULY 2012, FIGUEIRA DA FOZ, PORTUGAL, PUBLISHED BY IOP PUBLISHING FOR SISSA MEDIALAB, 2012. doi:10.1088/1748-0221/8/01/C01025

[10] Schilling, K., Design of Pico-Satellites for Education in Systems Engineering. In: IEEE Aerospace and Electronic Systems Magazine 21 (2006), S. 9-14, 2006 
[11] Schmidt, M., Ravandoor, K., Kurz, O., Busch, S., Schilling, K., Attitude Determination for the Pico-Satellite UWE-2. In: Space Technology 28. 2009, pp.67-74, 2009

[12] Schmidt, W. K. H., 1975, NucIM, 127, 285

[13] Švéda, L., et al., 2004, SPIE Proc. 5168, 393

[14] Tichý, V., et al., 2009, Balt. Astr. 18, 362

[15] Tichý, V., et al., 2011, Nucl. Instr. Meth. A, A633, S169

[16] Tichý, V., et al., 2013a, SPIE Proc. 8777, 877711

[17] Tichý, V., 2013b, SPIE Proc. 8777, 877710 